\title{
Preoperative patients' health decrease moderately, while hospital costs increase for hip and knee replacement due to the first COVID-19 lockdown in Germany
}

\author{
Caroline Schatz $^{1,2}$ (1) $\cdot$ Reiner Leidl ${ }^{1,2} \cdot$ Werner Plötz $^{3,4} \cdot$ Katharina Bredow $^{2} \cdot$ Peter Buschner $^{3}$ \\ Received: 10 November 2021 / Accepted: 23 January 2022 / Published online: 24 February 2022 \\ (c) The Author(s) under exclusive licence to European Society of Sports Traumatology, Knee Surgery, Arthroscopy (ESSKA) 2022
}

\begin{abstract}
Purpose The purpose of this study was a comparison between osteoarthritis patients with primary hip and knee replacements before, during and after the first COVID-19 lockdown in Germany. Patients' preoperative health status is assumed to decrease, owing to delayed surgeries. Costs for patients with osteoarthritis were assumed to increase, for example, due to higher prices for protective equipment. Hence, a comparison of patients treated before, during and after the first lockdown is conducted. Methods In total, 852 patients with primary hip or knee replacement were included from one hospital in Germany. Preoperative health status was measured with the WOMAC Score and the EQ-5D-5L. Hospital unit costs were calculated using a standardised cost calculation. Kruskal-Wallis tests and Chi-squared tests were applied for the statistical analyses.

Results The mean of the preoperative WOMAC Score was slightly higher $(p<0.01)$ for patients before the first lockdown, compared with patients afterwards. Means of the EQ-5D-5L were not significantly different regarding the lockdown status (NS). Length of stay was significantly reduced by approximately 1 day $(p<0.001)$. Total inpatient hospital unit costs per patient and per day were significantly higher for patients during and after the first lockdown $(p<0.001)$.

Conclusion Preoperative health, measured with the WOMAC Score, worsened slightly for patients after the first lockdown compared with patients undergoing surgery before COVID-19. Preoperative health, measured using the EQ-5D-5L, was unaffected. Inpatient hospital unit costs increased significantly with the COVID-19 pandemic.
\end{abstract}

Level of evidence Retrospective cohort study, III.

Keywords COVID-19 · Osteoarthritis · Joint replacement · Cost · Health-related quality of life

\section{Introduction}

The global outbreak of the corona-virus-pandemic (COVID-19) emphasised the importance of effective public health strategies all over the world [31]. In Germany, the

Caroline Schatz

schatz@bwl.lmu.de

1 LMU Munich School of Management, Institute of Health Economics and Health Care Management, LudwigMaximilians-Universität München, Munich, Germany

2 Institute of Health Economics and Health Care Management, Helmholtz Zentrum München, Munich, Germany

3 Krankenhaus Barmherzige Brüder München, Akademisches Lehrkrankenhaus der Technischen Universität München, Munich, Germany

4 Klinikum Rechts der Isar, Technical University Munich, Munich, Germany government pronounced the first nationwide socioeconomic lockdown on 16 March 2020. To prevent an overburdening of the health care system, the Ministry of Health requested all hospitals to delay their non-urgent surgeries. The postponement led to a reduction in total knee replacements (TKR) and total hip replacements (THR) for patients with osteoarthritis (OA). After the first lockdown, most restrictions were cancelled on 16 June.

The limited literature about COVID-19 and preoperative health, measured using patient-reported outcome measurements (PROM), indicated increasing pain for postponed patients, decreasing physical function and activity, and ambiguous results for mental health $[6,11]$. However, a small sample size study indicated increasing health-related quality of life (HRQoL) [14]. In hospitals, a wide range of measures was introduced to resume TKR and THR [12]. Literature about hospital unit costs was very scarce, and one study found that compensation payments by the government 
were not sufficient [29]. Hence, an investigation about the impact of COVID-19 on preoperative PROMs and costs was needed. To our knowledge, this is the first study analysing whether primary THR and TKR patients were affected in routine care, cross-sectional with 852 patients, covering a more comprehensive cohort than the subgroup of postponed patients. Preoperative patients' health serves as a predictor for postoperative outcomes and is, therefore, of high clinical relevance [28]. Furthermore, the EQ-5D is evaluated, additionally to the WOMAC. Similarly, to our knowledge, hospital unit costs for THR and TKR and COVID-19 were not investigated to date. These costs were important, because, in the short run, the retrospective diagnosis-related groups (DRG) system could not consider fast increasing unit costs. Accordingly, two hypotheses were developed.

H1: The mean of preoperative health, measured with the WOMAC, the EQ-5D and the EQ VAS, for patients with primary THR or TKR, is equal before, during and after the first COVID-19 lockdown.

H2: The mean of hospital unit costs for patients with primary THR or TKR is equal before, during and after the first COVID-19 lockdown.

\section{Methods}

The data were part of a project of the Munich Network Health Care Research-MobilE-Net, from one hospital, comprising 869 patients. Patients with OA of the hip or knee and a primary replacement of the joint were included, retrospectively. This cohort was classified into three groups. The first group included patients before the COVID-19 pandemic, from 5 August 2019 to 15 March 2020. The second group included patients during the lockdown period, from 16 March to 16 June, and the third group included patients between 17 June 2020 and the lockdown light, beginning on 1 November 2020.

\section{Patient characteristics}

Gender, date of birth and the indication of hip versus knee were collected from the clinical information system and the pain medication from the admission sheet. The indication, whether a patient received a hip or a knee replacement, was included as a dummy variable, to control for differences in these patient groups. A separate analysis of hips and knees was not performed, because the number of patients during the lockdown was too small. Age was calculated as the difference between the date of birth and the admission date, categorised below the mean of 70 years and above. Gender, indication, physical activity and pain medication were included as binary variables. The documentation of physicians was used to transform pre-illnesses into ICD-10 codes.
Then, the comorbidity indices of Elixhauser [5] and Charlson [2] with the adaptation to orthopaedics from Menendez [16] were calculated with the body mass index (BMI) included from medical records. For the anaesthesiological assessment, the ASA Score [20] was collected from the medical documentation.

\section{Patient-reported outcome measures (PROMs)}

Prior to the surgery, hospitalised patients answered the WOMAC questionnaire, the EQ-5D-5L including the EQ VAS, and the questions about physical activity. The WOMAC was transformed to a scale, ranging from 0 to 100 to allow comparisons with other publications, as suggested by Singh et al. [23].

\section{Cost calculation}

Hospital unit costs, initially calculated for the National Institute for the reimbursement system in Germany (InEK), were collected. This standardised cost calculation is a mixture of full costing with activity-based elements of inpatient costs at patient-level, where costs are calculated for an entire year, allocated to patients, with standardised allocation keys [27]. The three main cost components, ward, surgery and other costs were the basis for the cost analysis. Surgery costs included all costs related to the operating procedure including implants, technical equipment and personnel costs. Ward costs comprised all costs associated with treating the patient after surgery in a normal ward, including personnel costs, material costs and drugs. Other costs were the remaining costs, for example intensive care, radiology, laboratory and therapy. Overhead costs for the medical and non-medical infrastructure were allocated to all cost centres and, therefore, included in ward, surgery and other costs. Costs per day were calculated using the length of stay for each patient. Costs from 2019 were inflated by $0.5 \%$. Single missing items in the cost data were replaced with the mean of the entire cohort for two patients and one patient without cost data was deleted.

\section{Sample corrections}

From 869 patients, 1 with no cost data was deleted, 3 patients were omitted, because of inconsistent questionnaires, and another 3 were deleted because the primary diagnosis was not osteoarthritis. Missing values were found in EQ-5D-5L dimensions of usual activities (1 patient) and self-care (20 patients). These single-dimension missing items were replaced by the nearest value generating the patient's EQ VAS using the general population estimates of Leidl and Reitmeir [15]. Outliers were detected with boxplots, and extreme values above a threshold of twice the $75 \%$ 
quantile were deleted (10 patients). Patients with EQ VAS values of 0 were not deleted, because this phenomenon has already been discussed in the literature [21]. 852 patients remained in the analysis. The reporting of this study follows the STROBE checklist [30]. To account for potential biases, data collection and data analysis were done by two independent researchers, KB and CS. All patients, who were hospitalised with osteoarthritis and primary THR or TKR, within the given time periods, had an equal chance to be part of the study.

\section{Statistical analysis}

A correlation matrix was calculated to test multicollinearity between independent variables, with a threshold of $>0.7$ indicating a high correlation [4]. All correlations of control variables were below this threshold. The length of stay correlated highly $(>0.7)$ with the dependent variable of ward costs per patient and moderately with total cost per patient $(>0.5)$. Hence, the costs were calculated per patient and per day to include the length of stay into the dependent variable. PROMs were modelled as continuous variables. Homogeneity of variance among groups was tested with the Levene test statistic [9], and the normal distribution was examined with the Shapiro-Wilk test [22]. For continuous variables, the non-parametric test of Kruskal and Wallis [13], and, for the analysis of categorical variables, Chi-squared tests were used. The applied minimum significance level was $p<0.05$. The statistical analyses were done with $\mathrm{R}[8,10,18,19,26$, 32-34].

Table 1 Study cohort

\section{Results}

The description of the study cohort is depicted in Table 1 . During the lockdown, more male patients were treated, compared with the cohorts before and after. Physical activity remained stable during the lockdown but reduced afterwards. More severely ill patients, measured using the ASA Score, were treated during and after the lockdown, compared with patients before. Hence, the ASA Score differed significantly $(p<0.001)$ and physical activity moderately $(p<0.01)$, whereas almost no significant difference was found for gender $(p<0.05)$, and no statistical differences for comorbidities, age, pain medication and the indication of THR versus TKR (NS) (Table 1).

\section{Results' PROMs and costs}

The standardised WOMAC score increased for the group during the first lockdown and after the first lockdown. T. The main changes were found in pain $(p<0.01)$ and function $(p<0.01)$. Changes in WOMAC stiffness, but also in the EQ VAS and in the EQ-5D-5L dimensions were very small, resulting in non-significant results (NS) (Table 2).

Ward and surgery costs were identified as the main cost components responsible for approximately $80 \%$ of total costs. Total costs increased per patient during the first lockdown and after the first lockdown. Costs for surgery were almost stable, whereas ward costs rose for patients treated during and after the lockdown compared with patients before. The length of stay was reduced significantly by

\begin{tabular}{|c|c|c|c|c|c|c|c|c|c|c|}
\hline \multirow[t]{3}{*}{ 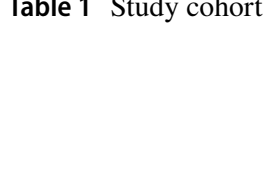 } & \multirow[t]{3}{*}{ Patient characteristics } & \multicolumn{3}{|c|}{ Entire cohort } & \multirow{2}{*}{\multicolumn{2}{|c|}{$\begin{array}{l}\begin{array}{l}\text { Before first } \\
\text { lockdown }\end{array} \\
(N=478)\end{array}$}} & \multirow{2}{*}{\multicolumn{2}{|c|}{$\begin{array}{l}\begin{array}{l}\text { During first } \\
\text { lockdown }\end{array} \\
(N=105)\end{array}$}} & \multirow{2}{*}{\multicolumn{2}{|c|}{$\begin{array}{l}\text { After first } \\
\text { lockdown }\end{array}$}} \\
\hline & & \multicolumn{3}{|c|}{$(N=852)$} & & & & & & \\
\hline & & $n$ & $(\%)$ & $p^{\mathrm{a}}$ & $n$ & $(\%)$ & $n$ & $(\%)$ & $n$ & $(\%)$ \\
\hline & Indication hip (THR) & 573 & $(67.3)$ & 0.630 & 315 & $(65.9)$ & 73 & $(69.5)$ & 185 & $(68.8)$ \\
\hline & Gender, male & 371 & $(43.5)$ & $0.016^{*}$ & 216 & $(45.2)$ & 55 & $(52.4)$ & 100 & $(37.2)$ \\
\hline & Physical activity, yes & 410 & $(48.1)$ & $0.007 * *$ & 247 & $(51.7)$ & 55 & $(52.4)$ & 108 & $(40.1)$ \\
\hline & Menendez index & & & 0.660 & & & & & & \\
\hline & $<0$ & 543 & $(63.7)$ & & 312 & $(65.3)$ & 61 & $(58.1)$ & 170 & $(63.2)$ \\
\hline & $=0$ & 177 & $(20.8)$ & & 93 & $(19.5)$ & 27 & $(25.7)$ & 57 & $(21.2)$ \\
\hline & $>0$ & 132 & $(15.5)$ & & 73 & $(15.2)$ & 17 & $(16.2)$ & 42 & $(15.6)$ \\
\hline & Age $<70$ years & 379 & $(44.5)$ & 0.684 & 214 & $(44.8)$ & 50 & $(47.6)$ & 115 & $(42.8)$ \\
\hline & Pain medication, yes & 317 & $(37.2)$ & 0.795 & 180 & $(37.7)$ & 41 & $(39.0)$ & 96 & $(35.7)$ \\
\hline & ASA Score & & & $<0.001 * * *$ & & & & & & \\
\hline & 1 & 113 & $(13.3)$ & & 82 & $(17.2)$ & 10 & $(9.5)$ & 21 & (7.8) \\
\hline & 2 & 591 & $(69.4)$ & & 331 & $(69.2)$ & 69 & $(65.7)$ & 191 & (71.0) \\
\hline & $3 / 4$ & 148 & $(17.3)$ & & 65 & (13.6) & 26 & $(24.8)$ & 57 & $(21.2)$ \\
\hline
\end{tabular}

Significance levels: $* 0.05, * * 0.01, * * *<0.001$

${ }^{\mathrm{a}}$ Chi-squared tests 
Table 2 Results' PROMs and costs

\begin{tabular}{|c|c|c|c|c|c|c|c|c|c|}
\hline & \multicolumn{3}{|l|}{ Entire cohort } & \multicolumn{2}{|c|}{ Before first lockdown } & \multicolumn{2}{|c|}{ During first lockdown } & \multicolumn{2}{|c|}{ After first lockdown } \\
\hline & \multicolumn{3}{|l|}{$(N=852)$} & \multicolumn{2}{|l|}{$(N=478)$} & \multicolumn{2}{|l|}{$(N=105)$} & \multicolumn{2}{|l|}{$(N=269)$} \\
\hline & Mean $\mp$ SD & Min-max & $p^{\mathrm{b}}$ & Mean $\mp$ SD & Min-max & Mean $\mp$ SD & Min-max & Mean $\mp$ SD & Min-max \\
\hline \multicolumn{10}{|l|}{ WOMAC } \\
\hline WOMAC score ${ }^{a}$ & $50.8 \mp 18.4$ & $0-100$ & $0.004 * *$ & $48.9 \mp 18.9$ & $0-100$ & $52.1 \mp 19.4$ & $0.4-89.5$ & $53.6 \mp 19.2$ & $5.4-100$ \\
\hline WOMAC pain & $10.1 \mp 4.0$ & $0-20.83$ & $0.002 * *$ & $9.6 \mp 3.9$ & 0-20.8 & $10.4 \mp 4.1$ & $0-20$ & $10.7 \mp 4.1$ & 0-20.8 \\
\hline $\begin{array}{l}\text { WOMAC stiff- } \\
\text { ness }\end{array}$ & $4.3 \mp 1.9$ & $0-8.33$ & 0.471 & $4.2 \mp 1.9$ & $0-8.3$ & $4.1 \mp 1.9$ & $0-8.3$ & $4.4 \mp 2.0$ & $0-8.3$ \\
\hline $\begin{array}{l}\text { WOMAC } \\
\text { function }\end{array}$ & $36.4 \mp 14.1$ & $0-70.83$ & $0.002 * *$ & $34.9 \mp 13.7$ & $0-70.8$ & $37.5 \mp 14.7$ & $0-64.5$ & $38.5 \mp 14.3$ & $1.6-70.8$ \\
\hline \multicolumn{10}{|l|}{ EQ VAS/EQ-5D-5L } \\
\hline EQ VAS & $57.0 \mp 21.7$ & $0-100$ & 0.509 & $57.9 \mp 20.8$ & $0-100$ & $55.3 \mp 23.3$ & $0-96$ & $56.1 \mp 22.6$ & $0-100$ \\
\hline EQ mobility & $3.1 \mp 0.9$ & $1-5$ & 0.207 & $3.0 \mp 0.8$ & $1-5$ & $3.1 \mp 0.9$ & $1-4$ & $3.1 \mp 0.9$ & $1-5$ \\
\hline EQ self-care & $1.8 \mp 0.9$ & $1-5$ & 0.549 & $1.8 \mp 0.9$ & $1-5$ & $1.9 \mp 0.9$ & $1-4$ & $1.9 \mp 1.0$ & $1-5$ \\
\hline EQ usual activity & $2.7 \mp 0.9$ & $1-5$ & 0.741 & $2.7 \mp 0.9$ & $1-5$ & $2.6 \mp 1.1$ & $1-5$ & $2.7 \mp 0.9$ & $1-5$ \\
\hline $\begin{array}{l}\text { EQ pain/discom- } \\
\text { fort }\end{array}$ & $3.2 \mp 0.8$ & $1-5$ & $0.019^{*}$ & $3.2 \mp 0.8$ & $1-5$ & $3.1 \mp 0.9$ & $1-5$ & $3.3 \mp 0.8$ & $1-5$ \\
\hline $\begin{array}{l}\text { EQ anxiety/ } \\
\text { depression }\end{array}$ & $1.7 \mp 0.9$ & $1-5$ & 0.524 & $1.7 \mp 0.8$ & $1-4$ & $1.7 \mp 0.8$ & $1-4$ & $1.8 \mp 0.9$ & $1-5$ \\
\hline \multicolumn{10}{|l|}{ Costs in Euro } \\
\hline $\begin{array}{l}\text { Costs total per } \\
\text { patient }\end{array}$ & $7125 \mp 1296$ & $3122-11,804$ & $<0.001$ & $6954 \mp 1317$ & $3122-11,654$ & $7276 \mp 1183$ & $5056-11,803$ & $7369 \mp 1260$ & $5017-11,752$ \\
\hline $\begin{array}{l}\text { Costs ward per } \\
\text { patient }\end{array}$ & $2900 \mp 735$ & $969-7223$ & $<0.001$ & $2811 \mp 713$ & $969-5353$ & $2991 \mp 717$ & $1017-4488$ & $3022 \mp 762$ & 1134-6089 \\
\hline $\begin{array}{l}\text { Costs surgery } \\
\text { per patient }\end{array}$ & $2967 \mp 691$ & $1236-6410$ & 0.191 & $2953 \mp 731$ & 1337-6409 & $2979 \mp 613$ & $1236-5481$ & $2985 \mp 646$ & 2133-6261 \\
\hline $\begin{array}{l}\text { Costs other per } \\
\text { patient }\end{array}$ & $1258 \mp 379$ & $646-5542$ & $<0.001$ & $1189 \mp 373$ & $649-5541$ & $1306 \mp 316$ & $772-2658$ & $1362 \mp 387$ & $741-4151$ \\
\hline $\begin{array}{l}\text { Costs total per } \\
\text { day }\end{array}$ & $1026 \mp 269$ & $265-2698$ & $<0.001$ & $932 \mp 202$ & 265-1933 & $1146 \mp 311$ & $739-2325$ & $1145 \mp 290$ & 297-2699 \\
\hline $\begin{array}{l}\text { Length of stay in } \\
\text { days }\end{array}$ & $7.3 \mp 2.0$ & $2-26$ & $<0.001$ & $7.7 \mp 1.8$ & $3-18$ & $6.7 \mp 1.9$ & $3-11$ & $6.8 \mp 2.3$ & $2-26$ \\
\hline
\end{tabular}

$S D$ standard deviation, mean arithmetic mean, min minimum, max maximum

Significance levels: $* 0.05, * * 0.01, * * *<0.001$

${ }^{\text {a}}$ WOMAC standardised from 0 to 100

${ }^{\mathrm{b}}$ Kruskal-Wallis tests

roughly 1 day $(p<0.001)$. Hence, the costs per day rose even more than the costs per patient. Costs measured per patient and per day were all significantly different $(p<0.001)$, except the costs for surgery (NS) (Table 2). The mean of total costs per patient (per day) increased by $2.8 \%(12.4 \%)$ for patients before the lockdown to during, and another $2.8 \%$ (12.4\%) after the lockdown (Table 3, Supplement).

\section{Sensitivity analyses}

The significance levels of all sensitivity analyses for total costs per patient did not change for the Kruskal-Wallis test. Although, the Kruskal-Wallis test statistic reduced the ward costs per patient for the third assumption from $p<0.001$ to $p<0.01$. Sensitivity analyses for costs per day were not performed, because the differences at base line are higher and hence the significance would not change, either. Robustness of results were further tested by additional sensitivity analyses, also including a multivariate GLM model, the results of which did not raise any robustness concerns (Table 3, Supplement).

For the PROMs, the EQ-5D-5L dimensions were defined as ordinal variables instead of continuous variables and, therefore, tested with Chi-squared tests. All dimensions were not significant, except for the pain dimension with a change in the significance level from $p<0.05$ to $p<0.01$. 


\section{Discussion}

The most important finding of this study was the moderate increase in pain and function, measured with the WOMAC Score, for patients with primary THR and TKR, and the simultaneously rising of costs per patient and per day during and after the lockdown, compared with patients treated before. These findings are generally in line with those of Endstrasser et al. [6], because differences for pain and function were found between the group before and after the lockdown, but not during. Although the effects were approximately half the magnitude, it should be considered that all patients undergoing TKR or THR were included in this study, not only the postponed ones. This delayed effect could be the reason for the improvement in HRQoL shortly after the lockdown, reported by Larghi et al. [14]. Increased pain was also found by Knebel et al. [11], but higher use of pain medication was not found. The dimension of anxiety/depression in the EQ-5D-5L was not significant, which strengthens the results from Endstrasser et al. [6] who found no influence on mental health and was partly in line with Knebel et al. [11] who found psychosocial distress only for middle-aged women. Given that the minimum clinically important difference (MCID) for the WOMAC Score ranging between 2 and 10 points (Siviero et al. [24], Clement et al. [3]), the 4.7 points found in this study is right in between, thus casting doubt on clinical relevance. Nevertheless, there may be a response shift towards perceived better outcomes, because TKR patients have been found to evaluate their HRQoL with a positive attitude just before surgery [7].

Implant costs as a main cost driver, reported by Stargardt [25], were found indirectly, because the implants were a major part of the surgery costs, whereas the COVID-19 pandemic had no significant impact on these costs. An explanation for stable surgery costs might be caused by the implants, because they are often goods on consignment, and without using them, no costs have to be paid. Although, there were additional costs, for example for COVID-19 tests and protective equipment, included in the costs for ward, and other costs. Furthermore, COVID19-positive patients might cause extra costs for special care and isolation on the ward with increased demands on nurses. The increased demands on personnel in general, for example because of quarantine, might have caused more temporary employment, with additional costs. The recommendations from Kort et al. [12] included a reduction in length of stay which was discovered in this study and leads to increased costs per day. The problem of idle capacity is well known in the field of cost accounting. Full-costing, top-down approaches cover these costs more adequately than solely activity-based ones [17]. The standardised cost calculation applied in this study was a mixture of both. Hence, costs for idle capacity were discovered, especially per day, where costs were allocated top-down not only to each patient but also for each day. Length of stay was highly correlated with ward costs, which was partly in line with Carducci et al. [1], but a correlation was also found for total costs and length of stay. The reason for this result was the calculation methodology of the costs, with days partly as allocation keys.

There are several limitations regarding the study design, the PROMs and the costs. The investigated lockdown status was not equal regarding the time period or the number of patients. This was determined as a result of the COVID19 pandemic and its lockdown. The smallest group consisted of 105 patients, to ensure robust results. This is also the reason why a classification of patients into hip and knee was not performed.

PROMs were collected during usual care with no consideration as to whether patients were on waiting lists, postponed the surgery voluntarily or had a planned surgery, as before the COVID-19 pandemic. Costs were evaluated for the study cohort of osteoarthritis with primary THR and TKR, considering neither the classification of DRGs nor the department structure of orthopaedics and trauma surgery. In addition, costs were compared in different seasonal settings, the cohort before COVID-19 in autumn and winter, the lockdown cohort in spring and the cohort after the lockdown in summer. Generally, the standardised cost calculation smoothed seasonal effects and elective THR and TKR were conducted equally across seasons, which reduced a potential bias. In addition, some seasonal effects probably remained, undistinguished from effects influenced by the COVID-19 pandemic, because this was itself a time-related variable.

All patients who met the criteria for the study and signed the consent form were included. Furthermore, the analysis took place in one hospital in Germany, deriving from a study on treatment outcomes and costs. This is a limitation to generalising the findings. Yet, this cost calculation approach could even be considered when the potential impact of idle capacity in TKR and THR is to be estimated, for example because of construction during day-to-day operation. Further research is necessary, especially for inpatient hospital costs, regarding the COVID19 pandemic for several hospitals, internationally, and in diverse surgical and non-surgical departments. In addition, the impact of a reduced level of preoperative WOMAC Score on postoperative results should be investigated given the predictive power of the preoperative values, and if possible, also the impact of reduced length of stay. 


\section{Conclusion}

The COVID-19 pandemic caused major disturbances in hospitals, but preoperative HRQoL changed only moderately for patients with OA and primary THR or TKR. Measured using the WOMAC Score, the means of pain and function were slightly different for patients before and after the COVID-19 pandemic. The EQ-5D-5L and the EQ VAS did not indicate significant differences. The COVID-19 pandemic influenced the inpatient hospital unit costs per patient and per day significantly, especially ward costs and other costs. Costs for surgery remained stable, whereas length of stay was reduced significantly by 1 day. Patient characteristics were almost similar, except for the increased number of patients with an ASA Score above 2. The clinical relevance for the WOMAC score was doubtful.

Supplementary Information The online version contains supplementary material available at https://doi.org/10.1007/s00167-022-06904-9.

Acknowledgements The authors would like to thank Ardit Gashi for feedback on an earlier draft of this article, Antonia Marx for the collection of medical data, Christoph Kurz for basic statistical support, the administrative staff of the participating hospital for providing cost data and Hadley Wickham and Matt Dowle for their R packages.

Author contributions CS: conceptualisation, data analyses and interpretation, and writing-main manuscript. RL: funding, supervision, and writing - review and editing. WP: supervision and writing-review and editing. KB: writing-main manuscript and data collection. PB: organisation of data, supervision, and writing — review and editing.

Funding This work is part of the project "Munich Network Health Care Research-MobilE-Net" and was supported by the German Federal Ministry of Education and Research Grant Number 01GY1603A.

\section{Declarations}

Conflict of interest The authors declare that they have no competing interests.

Ethics approval and consent to participate This study was approved by the ethics committee of Ludwig-Maximilians-Universität München (reference number:18-274). All included patients signed the consent to participate.

Consent for publication Not applicable.

Availability of data and materials Not applicable.

\section{References}

1. Carducci MP, Gasbarro G, Menendez ME, Mahendraraj KA, Mattingly DA, Talmo C et al (2020) Variation in the cost of care for different types of joint arthroplasty. J Bone Jt Surg Am 102:404-409
2. Charlson ME, Pompei P, Ales KL, MacKenzie CR (1987) A new method of classifying prognostic comorbidity in longitudinal studies: development and validation. J Chronic Dis 40:373-383

3. Clement ND, Bardgett M, Weir D, Holland J, Gerrand C, Deehan DJ (2018) What is the minimum clinically important difference for the WOMAC index after TKA? Clin Orthop Relat Res 476:2005-2014

4. Dormann CF, Elith J, Bacher S, Buchmann C, Carl G, Carré G et al (2013) Collinearity: a review of methods to deal with it and a simulation study evaluating their performance. Ecography $36: 27-46$

5. Elixhauser A, Steiner C, Harris DR, Coffey RM (1998) Comorbidity measures for use with administrative data. Med Care $36: 8-27$

6. Endstrasser F, Braito M, Linser M, Spicher A, Wagner M, Brunner A (2020) The negative impact of the COVID-19 lockdown on pain and physical function in patients with end-stage hip or knee osteoarthritis. Knee Surg Sports Traumatol Arthrosc 28:2435-2443

7. Felix J, Becker C, Vogl M, Buschner P, Plötz W, Leidl R (2019) Patient characteristics and valuation changes impact quality of life and satisfaction in total knee arthroplasty-results from a German prospective cohort study. Health Qual Life Outcomes 17:180

8. Gasparini, (2018) comorbidity: an R package for computing comorbidity scores. J Open Source Softw 3:648-651

9. Gastwirth J, Gel Y, Miao W (2010) The impact of Levene's test of equality of variances on statistical theory and practice. Stat Sci 24:343-360

10. Gastwirth JL, Gel YR, Wallace Hui WL, Lyubchich V, Miao W, Noguchi K (2020) lawstat: tools for biostatistics, public policy, and law. R package version 3.4. https://CRAN.R-project.org/packa ge=lawstat. Accessed 15 June 2021

11. Knebel C, Ertl M, Lenze U, Suren C, Dinkel A, Hirschmann MT et al (2021) COVID-19-related cancellation of elective orthopaedic surgery caused increased pain and psychosocial distress levels. Knee Surg Sports Traumatol Arthrosc 29:2379-2385

12. Kort NP, Barrena EG, Bédard M, Donell S, Epinette JA, Gomberg B et al (2020) Resuming elective hip and knee arthroplasty after the first phase of the SARS-CoV-2 pandemic: the European Hip Society and European Knee Associates recommendations. Knee Surg Sports Traumatol Arthrosc 28:2730-2746

13. Kruskal WH, Wallis WA (1952) Use of ranks in one-criterion variance analysis. J Am Stat Assoc 47:583-621

14. Larghi MM, Grassi M, Luca F, Placenza E, Rampulla C, Manzotti A (2020) Clinical outcome before and after COVID-19 quarantine in patients affect of knee and hip osteoarthritis. Acta Biomed 91:e2020150

15. Leidl R, Reitmeir P (2017) An experience-based value set for the EQ-5D-5L in Germany. Value Health 20:1150-1156

16. Menendez ME, Neuhaus V, Ring D (2015) Inpatient mortality after orthopaedic surgery. Int Orthop 39:1307-1314

17. Palsis JA, Brehmer TS, Pellegrini VD, Drew JM, Sachs BL (2018) The cost of joint replacement: comparing two approaches to evaluating costs of total hip and knee arthroplasty. J Bone Jt Surg Am 100:326-333

18. R Core $\mathrm{T}$ (2021) R: a language and environment for statistical computing. https://www.R-project.org/. Accessed 13 Aug 2021

19. Revelle W (2021) psych: procedures for personality and psychological research. https://CRAN.R-project.org/package=psych. Accessed 15 June 2021

20. Saklad M (1941) Grading of patients for surgical procedures. Anesthesiology 2:281-284

21. Scott CEH, MacDonald DJ, Howie CR (2019) "Worse than death" and waiting for a joint arthroplasty. Bone Jt J 101-b:941-950

22. Shapiro SS, Wilk MB (1965) An analysis of variance test for normality (complete samples). Biometrika 52:591-611 
23. Singh J, Sloan JA, Johanson NA (2010) Challenges with healthrelated quality of life assessment in arthroplasty patients: problems and solutions. J Am Acad Orthop Surg 18:72-82

24. Siviero P, Limongi F, Gesmundo A, Zambon S, Cooper C, Dennison EM et al (2019) Minimal clinically important decline in physical function over one year: EPOSA study. BMC Musculoskelet Disord 20:227

25. Stargardt T (2008) Health service costs in Europe: cost and reimbursement of primary hip replacement in nine countries. Health Econ 17:S9-20

26. Venables WN, Ripley BD (2002) Modern applied statistics with S. Springer, New York

27. Vogl M (2012) Assessing DRG cost accounting with respect to resource allocation and tariff calculation: the case of Germany. Health Econ Rev 2:15

28. Vogl M, Wilkesmann R, Lausmann C, Hunger M, Plötz W (2014) The impact of preoperative patient characteristics on health states after total hip replacement and related satisfaction thresholds: a cohort study. Health Qual Life Outcomes 12:108

29. von Dercks N, Körner C, Heyde C-E, Theopold J (2020) How badly is the coronavirus pandemic affecting orthopaedic and trauma surgery clinics?: an analysis of the first 5 weeks. Orthopade 49:494-501
30. von Elm E, Altman DG, Egger M, Pocock SJ, Gøtzsche PC, Vandenbroucke JP (2007) The Strengthening the Reporting of Observational Studies in Epidemiology (STROBE) statement: guidelines for reporting observational studies. Lancet 370:1453-1457

31. Xiang B, Li P, Yang X, Zhong S, Manyande A, Feng M (2020) The impact of novel coronavirus SARS-CoV-2 among healthcare workers in hospitals: an aerial overview. Am J Infect Control 48:915-917

32. Zeileis A (2006) Object-oriented computation of sandwich estimators. J Stat Softw 16:16

33. Zeileis A, Hothorn T (2002) Diagnostic checking in regression relationships. R News 2:7-10

34. Zeileis A, Köll S, Graham N (2020) Various versatile variances: an object-oriented implementation of clustered covariances in $\mathrm{R}$. J Stat Softw 95:1-36

Publisher's Note Springer Nature remains neutral with regard to jurisdictional claims in published maps and institutional affiliations. 